\title{
Mechanical characterization of strain-hardening cement based composites (SHCC) under impact shear load
}

\author{
Ahmed Tawfik ${ }^{1 *}$, Iurie Curosu ${ }^{1}$, and Viktor Mechtcherine ${ }^{1}$ \\ ${ }^{1}$ Institute of Construction Materials, Technische Universität Dresden, 01062 Dresden, Germany
}

\begin{abstract}
The paper presents a testing facility, which enables the characterization of the shear behavior of cementitious composites under quasi-static and impact shear load. The device was designed by means of an extensive numerical parameter study on device geometry, materials and wave propagation, as well as specimen configurations and boundary conditions. The new shear testing device was integrated into a gravity-driven Split-Hopkinson tension bar (SHTB) for shear impact experiments and in a hydraulic testing machine for quasi-static shear experiments on strainhardening cement-based composites (SHCC). The shear response of the SHCC specimens was derived from the force obtained on the transmitter bar in the SHTB accompanied by high-speed optical measurements, which also enabled an accurate analysis of the crack-opening displacements and fracture modes by means of Digital Image Correlation (DIC).
\end{abstract}

\section{Introduction}

Strain-hardening cement-based composites (SHCC) are a class of fiber reinforced composites consisting of fine-grained cementitious matrices and short, randomly distributed micro-fibers in a volume fraction usually ranging between $1 \%$ and $2 \%$ [1]. The most distinguished mechanical feature of SHCC is their high pre-peak strain capacity of up to $5 \%$, ensured by the development of multiple fine cracks under increasing tensile load [1]-[3]. The high tensile ductility along with the excellent crack control make SHCC suitable as thin strengthening layers in applications requiring high energy dissipation and damage tolerance [4]-[8]. However, the strengthening performance of SHCC does not only depend on their tensile properties but also on their shear behavior. Therefore, for a purposeful material design and formulation of design constitutive laws, material characterization of SHCC under quasi-static and dynamic shear load is required.

The shear behavior of SHCC has only been scarcely investigated due to the lack of simple and reliable shear testing methods at the material level. Established testing configurations for shear are punch through shear tests, anti-symmetric four-point bending and Z-type push-off experiments [9]-[14]. The investigation of the shear behavior of SHCC in anti-symmetric flexural tests yields fracture resulting from both flexural and shear crack propagation despite purposefully optimized notch geometries [15]. This makes the experimental derivation of the

*Corresponding author: ahmed.tawfik@tu-dresden.de 
shear resistance of SHCC challenging and requires numerical analysis additionally. Furthermore, the beam testing configurations are not suitable for dynamic shear testing due to the substantial inertia and structural effects. The punch through shear testing techniques using the split-Hopkinson pressure bar (SHPB) do not enable an optical monitoring of the fracture modes [16][17]. This disadvantage can be eliminated by using beam like specimens and suitable adapters such as in the strain energy frame impact machine (SEFIM) [18]. However, in this case the gripping and boundary conditions should be optimized for limiting the occurrence by flexural cracks and impose shear crack formation and fracture.

The latter principle was applied in the investigation by the authors presented in the paper at hand. The design principle assumed using the tensile loading wave in a split Hopkinson tension bar (SHTB) to induce shear loading into the specimen through tailored and optimized adapters [19][20]. The mechanical shear device to be integrated in the SHTB was designed by means of numerical modelling in order to assess the effect of the adapters on wave propagation and analyze the applicability of the wave analysis for deriving the specimen response. Furthermore, the numerical parameter study targeted the achievement of an adequate specimen configuration in terms of notches and boundary conditions. Quasi-static and impact shear experiments were conducted on SHCC and the specimen responses were analyzed in terms of shear resistance and fracture mode.

\section{Shear testing device}

The mechanical shear device was developed using Finite Element Modelling (FEM), the work was carried out in the FEM code LS-Dyna. The aim was to shape a device that could be integrated into an existing SHTB [19][20] and convert the generated tensile loading wave into shear loading in the specimen. A schematic of the SHTB and the developed mechanical shear device (adapters) are shown in Fig. 1. The SHTB consists of an input bar and transmitter bar, both made of brass and each with a diameter of $24 \mathrm{~mm}$. The tensile wave is generated when the steel strikers are allowed to drop under gravity onto the steel impact flange from a height of $0.65 \mathrm{~m}$, ensuring an impact velocity of $3.5 \mathrm{~m} / \mathrm{s}$.

The shear device had to ensure a shear fracture mode without distorting the wave propagation in the SHTB and at the same time allow optical crack monitoring in the loaded specimens. The optimization process of the shear adapters was carried out iteratively by removing the unnecessary mass from each adapter and shaping the geometry for reducing the impedance mismatch with the input and transmitter bars, respectively. At the same time, the material of the adapters must ensure a high stiffness and facilitate specimen failure without plastic deformations in the adapters themselves. For this reason, both adapters were made of stainless steel.

At each optimization step, the effect of adapters on the wave profile was verified. The resulting effects on wave propagation in the input and transmitter bars excluding the specimen are presented in Fig. 2a and b, next to the undistorted input loading wave. Evaluation of the waves was performed by comparing the reflected waves at the free ends of the bars without adapters to the reflected waves at the ends of the bars with rigidly attached adapters. The adapters were designed to be tightened to the bars through threaded connections. Locations 1 and 2 indicate where the first and the second wave reflections occur. The first reflection occurs when the wave first reaches the adapter and this reflection has the same sign as the input wave (tension), since the impedance of the adapters is higher than that of the bars. The second reflection occurs when the wave reaches the free end of the adapter and it has opposite sign to the input wave, i.e. compression. The adapters' geometries were optimized in such a way that the magnitudes of the first reflections are as low as possible and the second reflections match the waveform of the input wave. 
Fixation

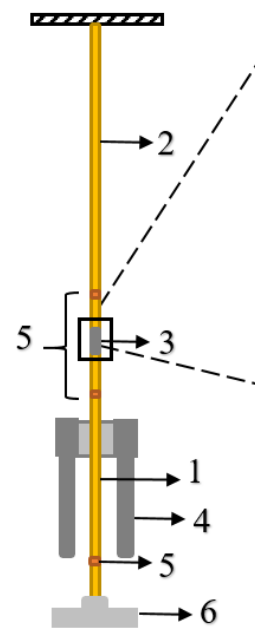

(a)

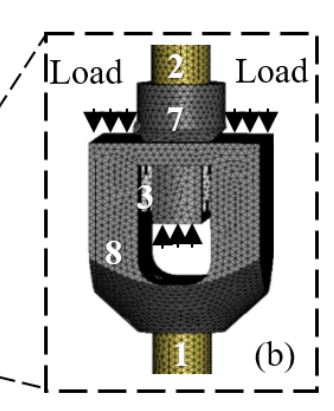

1) input bar $(1.31 \mathrm{~m})$

2) transmitter bar $(2.5 \mathrm{~m})$

3) specimen

4) steel strikers
$2 \mathrm{D}$ and $3 \mathrm{D}$ specimen views

$5 \mathrm{~mm}$ shear span

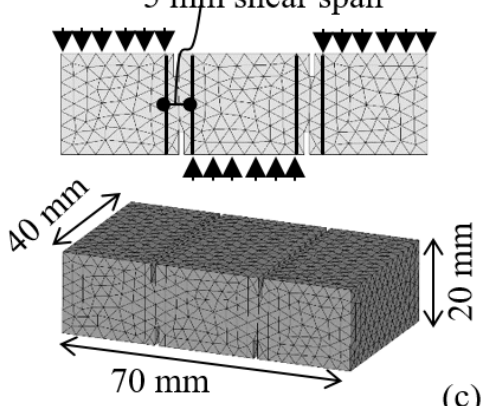

(c)

5) strain gauges

6) steel impact flange

7) upper shear adapter

8) lower shear adapter

Fig. 1. (a) SHTB schematic with (b) the integrated shear device and (c) specimen dimensions.

Fig. 2c shows that the reflected wave was not considerably affected by the upper adapter, since the tensile reflection is negligible and it shows the same profile as the input wave with no distortions. On the other hand, the tensile reflection due to the lower adapter was large enough to change the wave profile in comparison to the reference wave, making the mechanical measurements on the input bar inapplicable for deriving the specimen response through wave analysis. The reason for this is the substantially larger mass of the lower adapter, which increases the impedance mismatch between the steel adapter and the brass bar substantially.

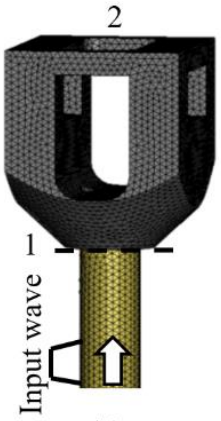

(a)

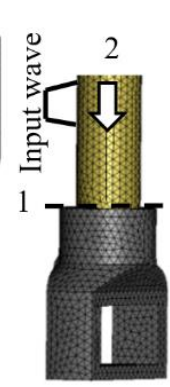

(b)

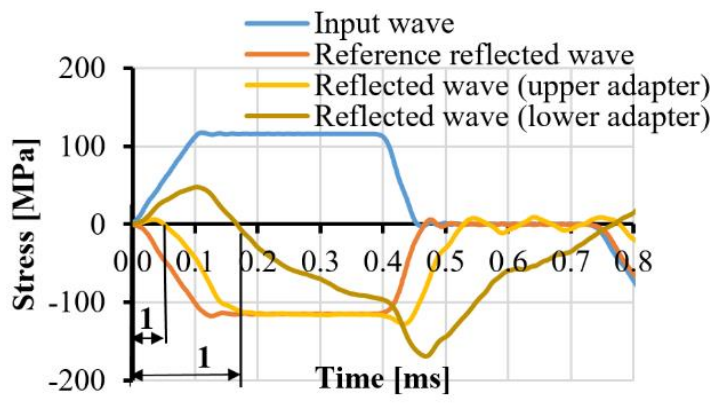

(c)

Fig. 2. Evaluation of the wave propagation through the (a) lower adapter and (b) upper adapter; (c) comparison of the input and reflected waves.

Upon reaching a satisfactory geometric solution for the loading adapters, the effect of lateral confinement and position of the notches within the shear span in the specimens were numerically investigated regarding their effect on the crack formation and fracture mode. Due to the sophisticated geometry of the shear device, the model was discretized with 4noded tetrahedron elements, with an element size of $3 \mathrm{~mm}$. The element formulation was defined based on a reduced integration scheme, such that each element has one integration point. All the simulations were conducted using the explicit solver of LS-Dyna. The time step 
was $8 \cdot 10^{-4} \mathrm{~ms}$, which satisfied the courant time step condition of stability in explicit time integration. The constitutive material model of the specimen in the numerical parameter study was the plain concrete model Karagozian \& case (K\&C) [21], while the bars were modelled as elastic materials with Young's modulus and Poisson's ratio corresponding to brass. The effect of confinement was investigated by applying two different contact types between the specimen and the shear adapters. The first contact type was a tied contact based on constraining or tying the specimen nodes to the nodes of the shear adapters, i.e., tied surface to surface contact. The second contact type allowed only compressive loads to be transferred between the nodes of the specimen and those of the shear adapters, that is, automatic surface to surface contact [22]. The first contact type is equivalent to gluing the specimen to the adapters, while the second type promoted a case with no confinement, allowing free lateral displacement (expansion) of the specimen.

The notch geometry and dimensions were also determined in preliminary numerical investigations and these were $1 \mathrm{~mm}$ wide and $5 \mathrm{~mm}$ deep. In this paper the effect of notch position within the $5 \mathrm{~mm}$-wide shear span is presented. In the default position case, the notches are positioned within the shear span at the edges of the loading adapters, as shown in Fig. 3a. In the second configuration the notches are shifted closer to each other, so that the inner edge of the upper notch coincides with the outer edge of the lower notch, leaving a distance of $1.5 \mathrm{~mm}$ between the edge of the notch and the edge of the loading adapters, as shown in Fig. 3b.

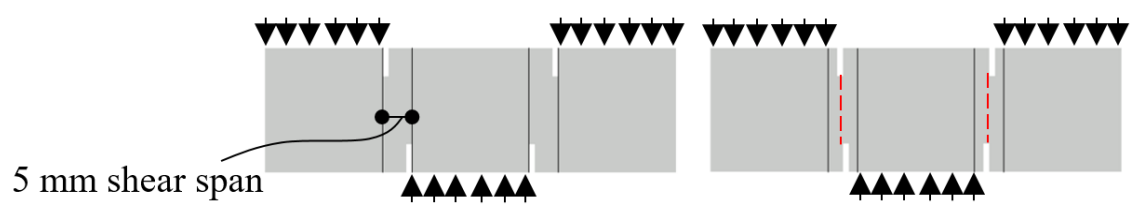

(a) Default position

(b) Shifted position

Fig. 3. (a) Default and (b) shifted notch configurations including the shear spans and loading principle; note that the boundary conditions are not presented.

\subsection{Experimental shear testing configurations}

Quasi-static shear experiments were carried out by incorporating the mechanical shear adapters in a hydraulic testing machine Instron 8501, USA. The adapters were fixed to threaded aluminum bars, which, at their turn, were clamped in the testing machine. The displacement rate was $0.05 \mathrm{~mm} / \mathrm{s}$. The deformations were monitored by a stereo optical system Aramis adjustable $12 \mathrm{M}$ by GOM and the acquired images were subsequently evaluated using DIC software GOM Correlate Professional, Germany [23]. The testing machine and the optical system were synchronized and this allowed deriving the forcedeformation histories.

In the impact shear testing setup, as shown in Fig. 1, strain gauges were glued on the input and transmitter bars to record the input, reflected and transmitted waves. However, given the strong distorting effect of the lower adapter on wave propagation, the force-time history was derived based only on the transmitted wave. The deformations were derived by using a highspeed stereo camera system consisting of two Photron SA-X2 480K-M1 cameras, provided by Photron Japan, with subsequent DIC analysis in the software GOM Correlate Professional, Germany [24][25].

Fig. 4 presents the shear testing device with the specimen fixation involving lateral confinement by gluing. Disregarding the boundary conditions in terms of lateral confinement, the gripping principle of the adapters does not allow specimen rotation. 


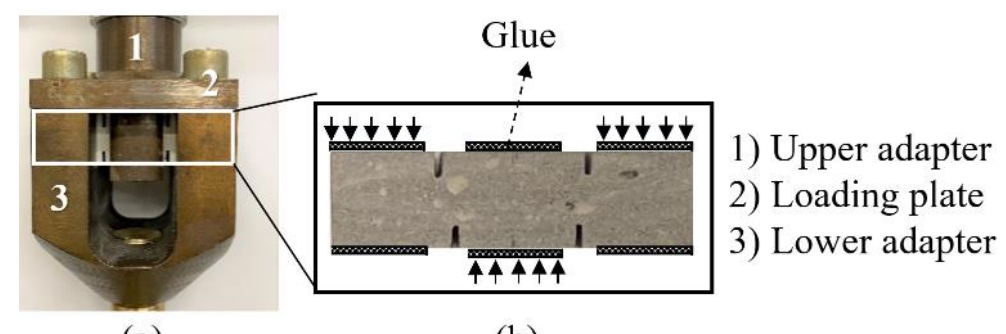

(a)

(b)

Fig. 4. (a) Assembled shear adapters with (b) applied boundary conditions in the shear experiments.

\section{SHCC composition}

The SHCC under investigation consists of a normal-strength cementitious matrix and $6 \mathrm{~mm}$ long fibers made of ultra-high molecular weight polyethylene (short PE). The cementitious matrix is typical for normal-strength SHCC and it is characterized by a high volume of cement and fly ash, a relatively low content of fine aggregates and a water-to-cement ratio of 0.674 , which can be explained by the high content of fines; see Table 1 . The fibers are produced by DSM, the Netherlands, under the brand name Dyneema SK60 [26]. The PE fibers have an average diameter of $18 \mu \mathrm{m}$, a tensile strength of $2500 \mathrm{MPa}$, and Young's modulus of $80 \mathrm{GPa}$. They are characterized by their hydrophobicity which induces a relatively weak frictional bond with the cementitious matrix.

Table 1. SHCC mixture composition.

\begin{tabular}{lc} 
& {$\left[\mathbf{k g} / \mathbf{m}^{\mathbf{3}}\right]$} \\
\hline CEM I 52.5 R-SR3/NA (Holcim, Germany) & 506 \\
\hline Fly ash (Steag Power Minerals, Germany) & 620 \\
\hline Quartz sand 0.06/0.2 mm (Strobel Quarzsand, Germany) & 536 \\
\hline Viscosity modifying agent (SIKA, Switzerland) & 2.0 \\
\hline UHMWPE fiber 2.06 \% by volume (DSM, the Netherlands) & 20 \\
\hline High-range water reducing agent, Glenium ACE 460 (BASF, Germany) & 11 \\
\hline Water & 341
\end{tabular}

\section{Results and discussion}

\subsection{Impact simulations and numerical parameter study}

The effect of specimen confinement against lateral deformation as derived in numerical simulations is presented in Fig. 5. The curves show the transmitted force by the specimen relative to the lateral deformation without and with lateral confinement, respectively. The lateral deformation plotted represents the deformation (expansion) of one side of the specimen. Confining the specimen restricted their lateral movement and resulted in a considerably higher force transmitted by the specimen and more localized shear stresses in the ligament compared to the case with no lateral confinement. Therefore, applying confinement and suppressing the overall dilation of the specimen enhances the apparent shear resistance and is an essential condition to avoid tensile fracture mode. Similar interpretations were also reported in [10][16][17]. 

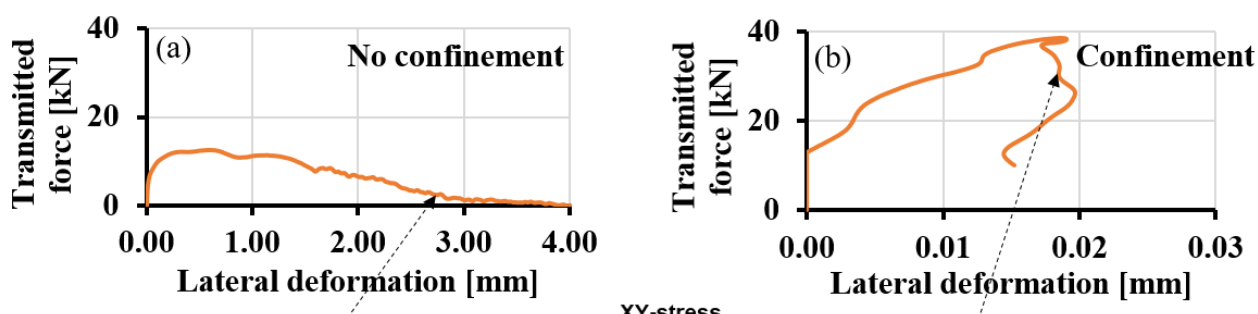

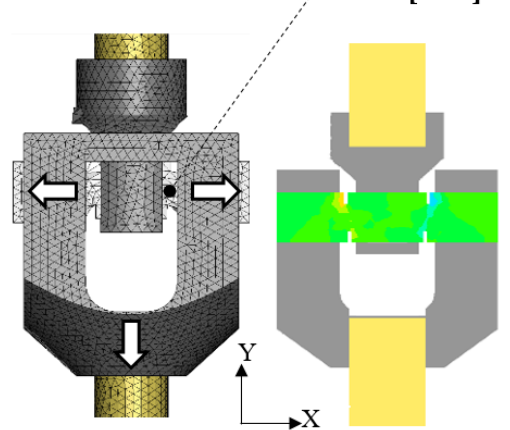

(c)

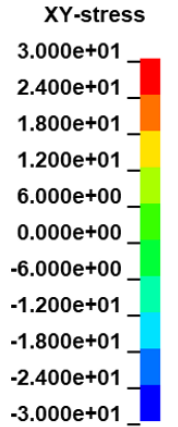

$-3.000 \mathrm{e}+01$

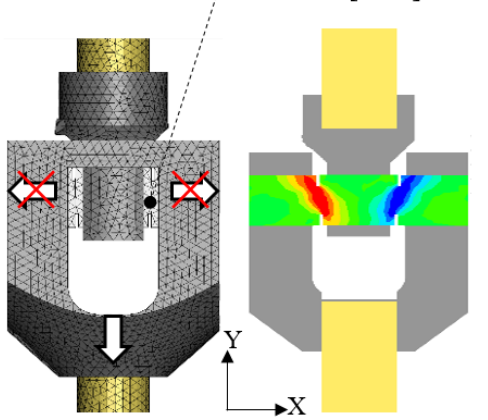

(d)

Fig. 5. Transmitted force relative to lateral deformation for (a) unconfined and (b) confined samples. Shear stress ( $\mathrm{x}-\mathrm{y})$ for $(\mathrm{c})$ unconfined and (d) confined samples at the time of failure, i.e. peak load.

In Fig. 6, the effect of the notch position is presented only for the confined configuration. The curves show the transmitted force relative to the vertical deformation of the sample. The default position case showed a slightly higher transmitted force than in the shifted case. Although in both cases the shear span is $5 \mathrm{~mm}$, the shear zone appeared to be larger and with a slightly larger inclination angle in the default case than in the shifted case. The shear crack is expected to propagate in the shear zone between the upper and lower notches. Therefore, in the default case, where the horizontal distance between the inner edges of the upper and lower notches is $3 \mathrm{~mm}$, the crack developing in the shear zone would develop with a higher inclination angle, promoting a mixed mode of failure with a higher influence of mode I. Shifting the notches towards each other promotes a failure governed more by mode II with a more vertically oriented shear crack. The idea that a narrower shear force zone would support a vertically propagating shear crack was also shown in [12].

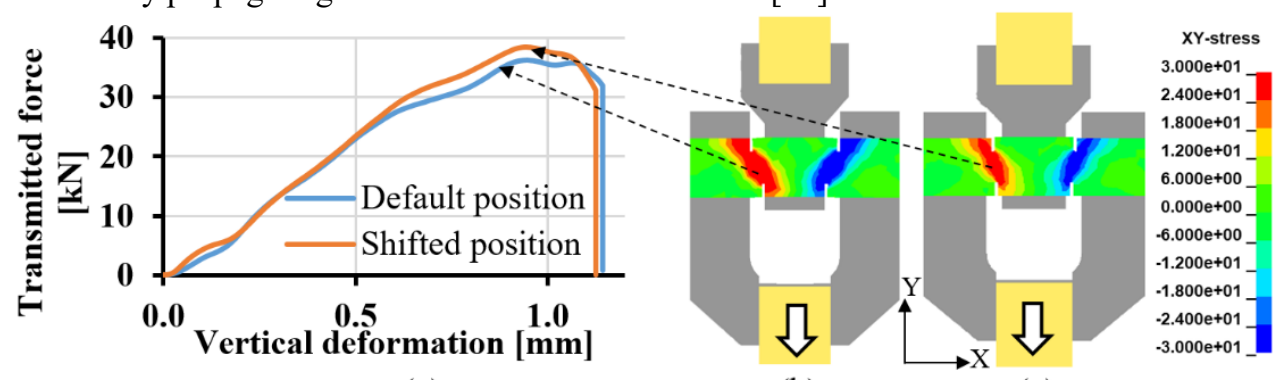

(a)

(b)

(c)

Fig. 6. Transmitted force relative to vertical deformation for both notches positions; shear stress (x-y) in (b) default position and (c) shifted position notches.

\subsection{Experimental results}

Based on the numerical results presented in Section 4.1, shear experiments were conducted on the SHCC specimens with shifted notch positions and with confinement. For practical 
purposes and easiness of the test preparation, the lower shear adapter was fabricated as two pieces connected with bolts; lower part and loading plate, as shown in Fig. 4. Full confinement was provided to the specimens by gluing the faces in contact with the shear adapters with a bi-component epoxy adhesive.

\subsubsection{Quasi-static shear tests}

Fig. 7 presents the transmitted force relative to the vertical deformation of an SHCC sample, as well as the force relative to mode II crack opening displacement (COD) of the shear crack flanks in both shear spans, based on the wave recordings in the transmitter bar synchronized with the DIC frames. LSS and RSS indicate the left and right shear spans, respectively. The vertical deformation of the sample was derived by tracking the distance between predefined markers inside the specimen throughout the test (pt.2-pt.1), as shown in Fig. 8b. Calipers were placed on the crack flanks in order to track mode II COD (pt.2'-pt.1') parallel to the yaxis, see Fig. 8d. The later calipers exclude the tensile cracks. The difference between the vertical deformation obtained for the sample and mode II COD is actually due to the formation of the tensile cracks (mode I) in both shear spans, prior to shear deformation (mode II). This can be seen in the failure process of the SHCC sample as obtained from DIC. At the time frames $0 \mathrm{~s}$ and $2 \mathrm{~s}$, the force increases linearly with the deformation until inclined tensile cracks form from the inner edges of the top notches and outer edges of the bottom notches; see Fig. 7. At the time frames between $9.5 \mathrm{~s}$ and $11.5 \mathrm{~s}$, several inclined cracks form in the middle of the LSS and finally merged into a shear crack connected from the outer edge of the top notch and the inner edge of the bottom notch. Maximum force was reached after $14 \mathrm{~s}$, at which a shear crack has already formed in the LSS, while several inclined cracks formed in the RSS. Finally, at a time of $17.5 \mathrm{~s}$ is the post-peak stage, where both shear spans yield extensive shear displacements (mode II). Virtual calipers were also used for measuring mode I COD of the tensile and shear cracks forming in both shear spans as shown in Fig. 8c and plotted in Fig. 9a. It is clear how the tensile cracks form before the shear cracks. Therefore, the sample failure is caused by initiation of tensile cracks which are arrested later, followed by failure due to a shear crack. Those initial tensile cracks were also found in [10][17]. To verify the reproducibility of the tests and failure modes, 5 samples were tested and the results are plotted in Fig. 10a and summarized in Table 2.

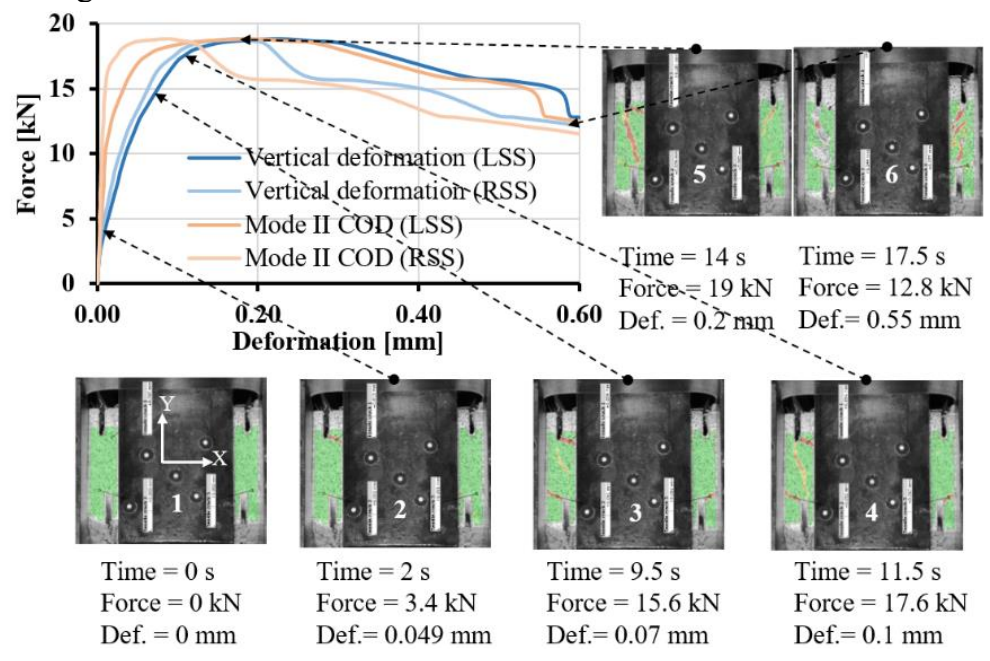

Fig. 7. Force relative to vertical deformation of the specimen synchronized with DIC frames (blue curves) and force relative to mode II COD (brown curves) from a quasi-static shear experiment on an SHCC sample. 


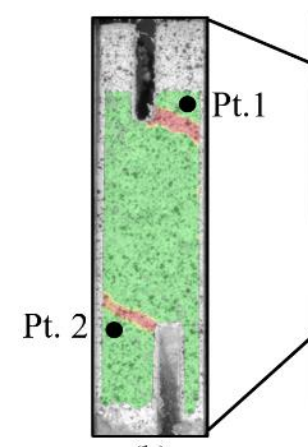

(b)

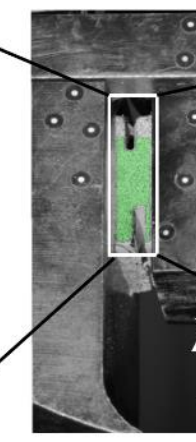

(a)

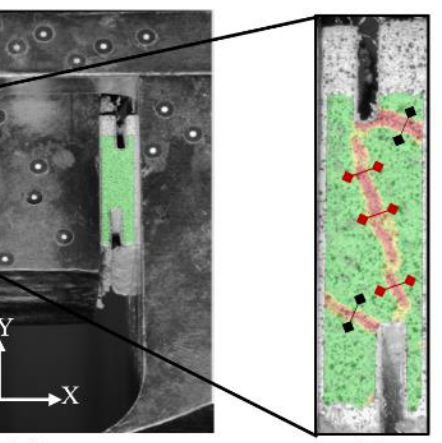

(c)

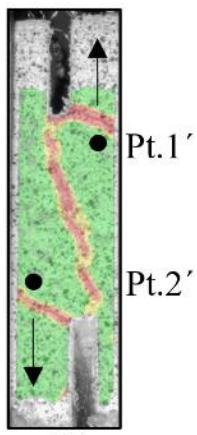

(d)

Fig. 8. (a) Shear device with the sample, (b) positions of the virtual calipers for deriving the sample's vertical deformation in the shear span, (c) calipers around the tensile cracks and shear crack to obtain mode I COD and (d) calipers positions for obtaining mode II COD of the shear crack.
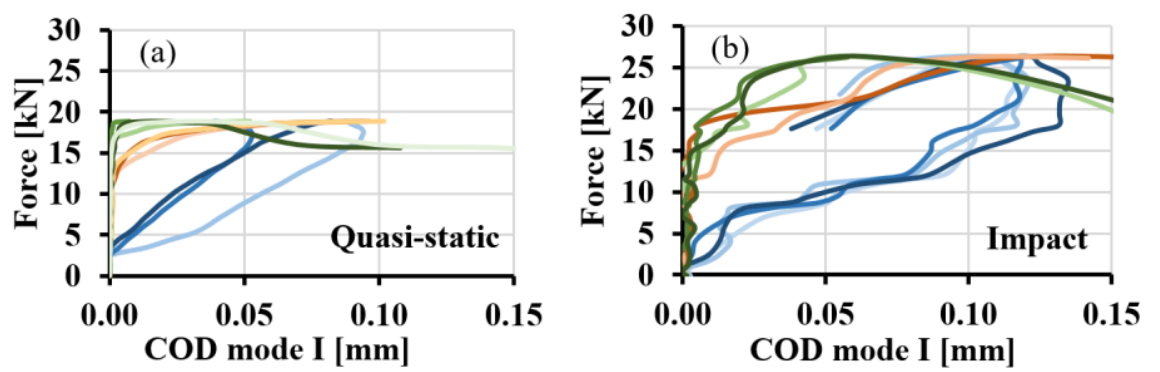

Fig. 9. Force relative to COD mode I for tensile and shear cracks developed in both shear spans for (a) quasi-static and (b) impact shear experiments on SHCC samples (blue curves indicate tensile cracks, red curves indicate shear cracks in the left shear span and green curves indicate shear cracks in the right shear span).
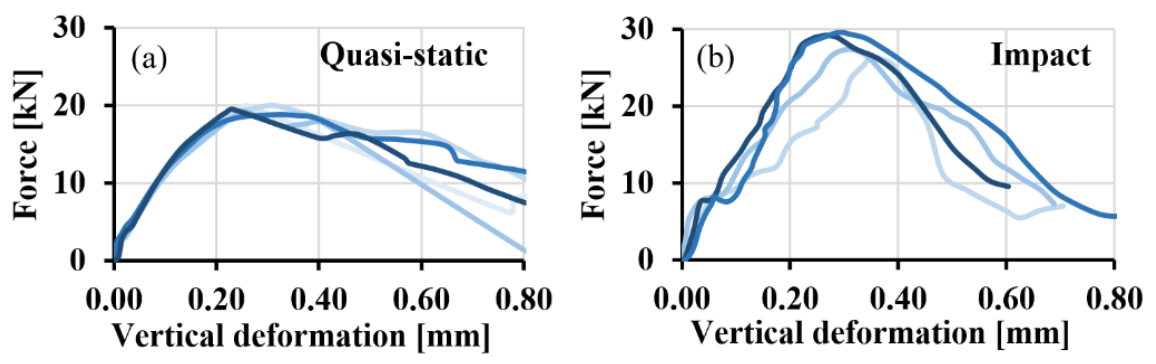

Fig. 10. Transmitted forces relative to the vertical deformations of SHCC specimens in (a) quasi-static and (b) impact shear experiments.

\subsubsection{Impact shear tests}

Fig. 11 presents the transmitted force relative to the vertical deformation of an SHCC sample, as well as the force relative to mode II crack opening displacement (COD) in both shear spans obtained in an impact experiment. Like quasi-static tests, both vertical deformation of the specimen and COD mode II were derived using DIC analysis as explained in Fig. 8. The fracture behavior is similar to the quasi-static tests and is shown by DIC frames synchronized with the force relative to vertical deformation curve, see fig. 11. The curve starts in a linear pattern from stage 1 until stage 2, where tensile cracks form at the top and bottom notches, similar to quasi-static tests. Then, in stages 3 and 4, a shear crack is connected, in the LSS, between the top and bottom notches, after several inclined cracks have formed. In stage 5 , 
the peak force is reached and a shear crack, similar to the LSS, forms in the RSS. Finally, stage 6 is the post peak softening stage.

The difference between the vertical deformation of the specimen and mode II COD is more pronounced in case of impact loading. This means that the COD mode I of the initial tension cracks are larger in impact than in the quasi-static case. This can be seen in Fig. 9, at which COD mode I for the tensile and shear cracks formed in both shear spans are compared for both loading regimes. A similar fracture behavior was reproduced for a set of SHCC samples tested under impact load, see Fig. $10 \mathrm{~b}$ and Table 2. In comparison to quasi-static tests, the initial tensile cracks (first cracking) formed at a higher force under impact load, with a dynamic increase factor (DIF) of 2 , while a DIF of 1.5 was obtained for the peak force.

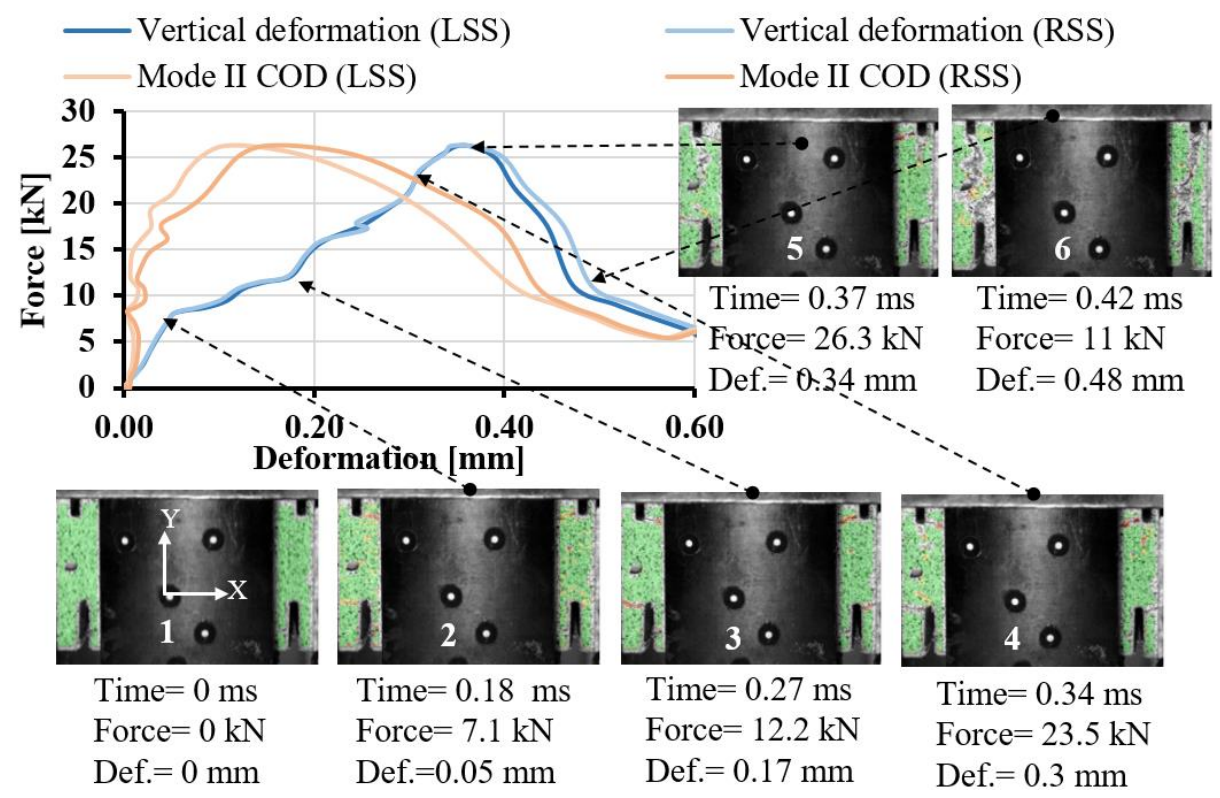

Fig. 11. Transmitted force relative to vertical deformation synchronized with DIC frames and force relative to mode II COD from impact shear experiments on an SHCC sample.

Table 2. Summary of quasi-static and impact shear experiments; average values, standard deviations are given in parentheses.

\begin{tabular}{ccccc} 
& \multicolumn{2}{c}{ Transmitted force $[\mathbf{k N}]$} & \multicolumn{2}{c}{ Vertical deformation $[\mathrm{mm}]$} \\
\hline & First crack & Peak & First crack & Peak \\
\hline QS & $3.7(0.4)$ & $19.5(0.4)$ & $0.02(0.00)$ & $0.28(0.04)$ \\
\hline Impact & $7.6(0.3)$ & $28.1(1.3)$ & $0.05(0.01)$ & $0.31(0.03)$
\end{tabular}

\section{Conclusions}

A mechanical shear device consisting of two adapters was designed, fabricated and integrated into a SHTB. The wave propagation in the impact shear experiments was distorted by the lower adapter attached to the input bar due to its considerable mass, making the wave analysis for deriving the specimen response invalid. For this reason, the assessment of the specimen response in terms of force-deformation relationships could be only done with auxiliary optical measurements combined with the recorded transmitted force in the transmitter bar. 
The optical measurements and DIC analysis allowed also a detailed analysis of the crack opening modes in the loaded specimens. The fracture modes of the confined SHCC samples in both regimes were characterized by the initial formation of tensile "wing" cracks at the locations of the maximum principle stresses. The tensile cracks were later arrested and the final shear fracture resulted from the interconnection of multiple cracks in the shear spans. The notches geometry and the boundary conditions of the specimen have to be further numerically investigated, in order to limit mode I cracks prior to mode II failure. Furthermore, the numerical simulations will help distinguish the structural and inertia effects from the actual specimen response under load.

\section{References}

[1] V. C. Li, J. Adv. Concr. Technol., $1215-230$ (2003)

[2] V. Li, S. Wang, C. Wu, ACI Mater. J., 98 483-492 (2001)

[3] I. Curosu, M. Liebscher, V. Mechtcherine, C. Bellmann, S. Michel, Cem Concr Res, 98 71-81 (2017)

[4] M. Maalej, S. T. Quek, S. F. U. Ahmed, J. Zhang, V. W. J. Lin, K. S. Leong, Constr Build Mater., 36 216-227 (2012)

[5] M. D. Lepech, V. C. Li, Mater. Struct., 42 1185-1195 (2009)

[6] M. Maalej, V. W. J. Lin, M. P. Nguyen, S. T. Quek, Eng. Struct., 32 2432-2439 (2010)

[7] V. C. Li, M. Lepech, Transportation research board 83rd annual meeting, Washington DC, USA (2003)

[8] S. Pourfalah, D. M. Cotsovos, B. Suryanto, M. Moatamedi, Eng. Struct., 173 10021018 (2018)

[9] T. Soetens, S. Matthys, Cem Concr Compos, 82 1-13 (2017)

[10] T. Backers, O. Stephansson, Rock Mech Rock Eng, 45 1011-1022 (2012)

[11] S. E. Swartz, L. W. Lu, L. D. Tang, T. M. E. Refai, Exp. Mech., 28 146-153, (1988)

[12] Z. P. Bažant and P. A. Pfeiffer, Mater. Struct., 19 111-121, (1986)

[13] T. Thuong, J. Kil, S. Pyo, D. Joo, Constr Build Mater., 151 246-257 (2017)

[14] J. Watkins, Int. J. Fract., 23 1983-1986 (1983)

[15] Q. Shang, G. P. A. G. Van zijl, J. S. Afr. Inst. Civ. Eng., 49 16-23 (2007)

[16] R. Abdul Rahman, Université Grenoble Alpes (2018)

[17] B. Lukić, P. Forquin, Int. J. Impact Eng., 91 34-45, (2015)

[18] T. T. NGO, D. J. KIM, Int. J. Impact Eng., 111 187-198, (2018)

[19] A. A. Heravi, I. Curosu, V. Mechtcherine, Cem Concr Compos, 105 (2019)

[20] A. Heravi, V. Mechtcherine, $10^{\text {th }}$ international conference on fractures mechanics of concrete and concrete structures, 23-26 June, Bayonne, France (2019)

[21] Livermore Software Technology Corporation (LSTC), LS-Dyna keyword user's manual Volume 1, (2014)

[22] U. Stelzmann, “2012_Explizit_Kontakte_UM," ANSYS Conf. 30 CADFEM Users Meet., 2012

[23] GOM Optical Measuring Technologies, User Manual - Hardware, (2009)

[24] Photron limited, Fastcam SA-X2 hardware manual, 1-60 (2019)

[25] GOM Optical Measuring Technologies, Photron Produktinformation, 1-52 (2015)

[26] Gruschwitz Textilwerke AG, Ultra High Molecular Weight Polyethylene Fiber from DSM Dyneema, 49 1-4 (2016) 